\title{
New Cellulose-Supported Reagent: a Sustainable Approach to Guanidines
}

\author{
Andrea Porcheddu, "Giampaolo Giacomelli, Alessandra Chighine, and Simonetta \\ Masala \\ Dipartimento di Chimica, Università degli Studi di Sassari, Via Vienna 2, I-07100 Sassari, \\ Italy
}

\section{Supporting Information}

General Methods. All reagents and solvents were obtained from Aldrich, Avocado or Acros Organics. Perloza AV-100, particle size 100-250 mm was obtained from Iontosorb (Czech Republic); loading of aniline bead cellulose is about $0.5 \mathrm{mmol} / \mathrm{g}$. Commercial bead cellulose is supplied as pre-swollen (water) gel. Cellulose beads were agitated using standard laboratory shakers or roller mixers. All reagents and solvents employed were reagent grade materials purified by standard methods and distilled before use. All conventional reactions were run under dry nitrogen in flamedried glassware using standard techniques unless otherwise stated. Thin-layer chromatography (TLC) analysis was performed with Merck Kieselgel 60 F254 plates and visualized using UV light, $\mathrm{KMnO}_{4}$ staining and the Sakaguchi reagent (spray of a $0.1 \%$ solution of 8 -hydroxyquinoline in acetone followed by a spray of a mixture of

\footnotetext{
* To whom correspondence should be addressed. E-mail: anpo@uniss.it
} 
$0.2 \mathrm{~mL}$ of bromine in $100 \mathrm{~mL}$ of $0.5 \mathrm{~N} \mathrm{NaOH}$ ). Melting points were determined in open capillary tubes on a Buchi apparatus and are uncorrected. The experiments were performed using using a flask equipped with a reflux condenser mounted outside the apparatus. FT-IR spectra were recorded as $\mathrm{KBr}$ pellets on Bruker Equinox 55 spectrometer (value in $\mathrm{cm}^{-1}$ ). Low resolution electronspray mass spectrometry was performed using VG Quattro (quadrupole-hexapole-quadrupole, QHQ) mass spectrometer system (Fisons Instruments, VG Analytical; Manchester, UK). Analytical HPLC was performed using a reversed phase Nucleosil C18 $(5 \mu \mathrm{m})$ column (250 x $2 \mathrm{~mm})$ (Grom, Herremberg, Germany). Solvents used for HPLC elution were (A) $\mathrm{H}_{2} \mathrm{O}$ containing $0.1 \%$ TFA, (B) $95 \%$ acetonitrile in $\mathrm{H}_{2} \mathrm{O}$ containing $0.1 \%$ TFA. The column was eluted with a linear gradient of solvent A going to $21 \%$ solvent B in A over the course of $5 \mathrm{~min}$ at a flow rate of $1.0 \mathrm{~mL} / \mathrm{min}\left(220 \mathrm{~nm}\right.$ detection). ${ }^{1} \mathrm{H}$ NMR (300 MHz) and proton-decoupled ${ }^{13} \mathrm{C}$ NMR (75.4 MHz) Fourier transform spectra were obtained with Varian VXR-300 spectrometer in $\mathrm{CDCl}_{3}$ using $\mathrm{CDCl}_{3}$ solutions and TMS as an internal standard. Chemical shifts are expressed in ( $\delta)$ ppm values, and coupling constants are expressed in Hertz $(\mathrm{Hz})$. The following abbreviations are used: $\mathrm{s}=$ singlet, $\mathrm{d}=$ doublet, $\mathrm{m}=$ multiplet and brs $=$ broad singlet. Elemental analyses were performed on a Perkin-Elmer 420 B analyzer. 
<smiles>N=C(N)n1cccn1</smiles>

1H-Pyrazole-1-carboxamidine Hydrochloride (1). To pyrazole (8.17 g, $0.12 \mathrm{~mol})$ and cyanamide $(5.05 \mathrm{~g}, 0.12 \mathrm{~mol})$ in $p$-dioxane $(120 \mathrm{~mL})$ was added $4 \mathrm{~N} \mathrm{HC1}(31$ $\mathrm{mL}$ ) in $p$-dioxane. The mixture was gently refluxed with stirring for $2 \mathrm{~h}$ under nitrogen. During the course of the reaction the product crystallizes. After cooling to room temperature, anhydrous ether $(30 \mathrm{~mL})$ was added and the mixture allowed to stand for $30 \mathrm{~min}$. The white crystalline product was collected by filtration, washed with anhydrous ether, and dried to constant weight in vacuo to yield $15.8 \mathrm{~g}(90 \%)$ : $\mathrm{mp}$ 164-165 ${ }^{\circ} \mathrm{C}$ (lit. $\left.{ }^{1} \mathrm{mp} 165-66{ }^{\circ} \mathrm{C}\right) ;{ }^{1} \mathrm{H}$ NMR (DMSO-d $\left.{ }_{6}\right) \delta(p p m): 9.77$ (brs, 4H), 9.06 $(\mathrm{d}, 1 \mathrm{H}), 8.12(\mathrm{~s}, 1 \mathrm{H}), 6.85(\mathrm{t}, 1 \mathrm{H}) .{ }^{13} \mathrm{C}$ NMR $\left(\mathrm{DMSO}_{-} \mathrm{d}_{6}\right) \delta(\mathrm{ppm}): 153.2,146.1,132.2$, 112.4. (ESI + ve ion): $111.1\left[(\mathrm{M}+\mathrm{H})^{+}\right]$.

\section{Colorimetric assay:}

$\beta$-Naphthol test. A few beads of the cellulose were successively washed with DMF and $\mathrm{MeOH}$. To a small portion $(5 \mathrm{mg})$ of cellulose in $\mathrm{MeOH}(1 \mathrm{~mL})$ at $0^{\circ} \mathrm{C}, 0.2 \mathrm{~mL}$ water-solution of $\mathrm{NaNO}_{2}(2 \%)$ and $0.2 \mathrm{~mL}$ water-solution of $\mathrm{HCl}(2.5 \mathrm{~mol} / \mathrm{l})$ were gradually added. After 1 min the activated support was separated and rinsed with $\mathrm{H}_{2} \mathrm{O}$ $(4 \times 3 \mathrm{~mL})$. To a filtered off support $0.3 \mathrm{~mL}$ aqueous solution of $\beta$-naphthol $(1 \% \mathrm{in}$ $\mathrm{NaOH} 1 \mathrm{~N}$ ) was added and the mixture was shaken for $30 \mathrm{sec}$ and the beads rinsed

(1) Bredereck, H.; Effenberger, F.; Hajek, M. Chem. Ber. 1965, 98, 3178. 
several times with a phosphate buffer $(0.1 \mathrm{~mol} / \mathrm{l}), \mathrm{H}_{2} \mathrm{O}$ and $\mathrm{MeOH}$. If free primary aromatic amino groups are present on the solid support the beads turn red. It is recommended that for each test a blank experiment be run in parallel.

\section{Guanidines synthesis}
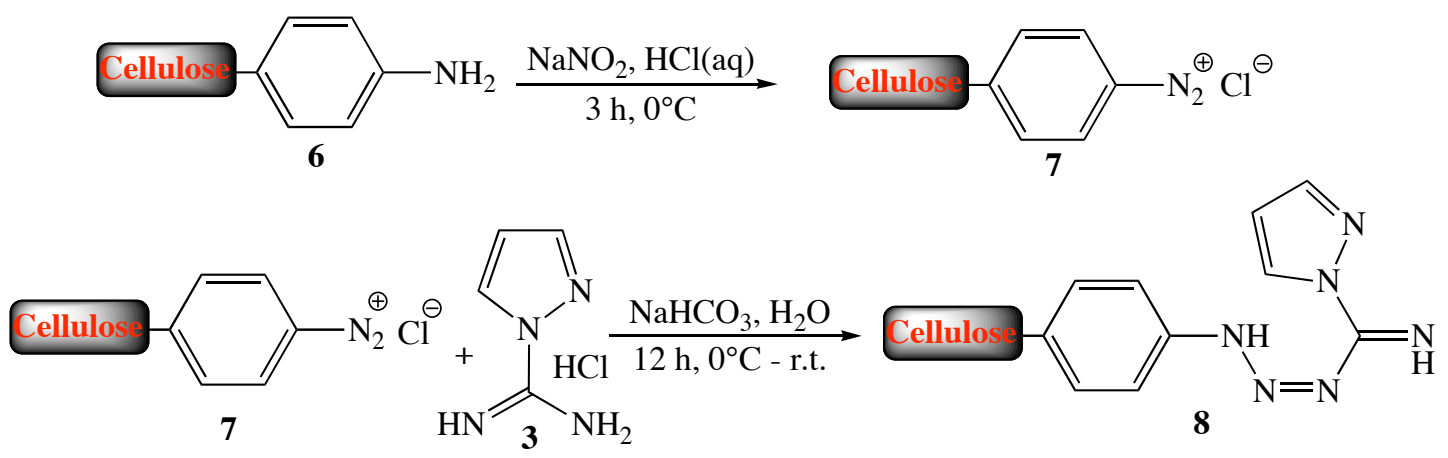

\section{Preparation of solid supported reagent 8}

Aniline Cellulose 6 ( $2 \mathrm{~g}, 1 \mathrm{mmol})$ was added to a chilled solution of $\mathrm{NaNO}_{2}(0.21 \mathrm{~g}$, $3.0 \mathrm{mmol})$ in $\mathrm{H}_{2} \mathrm{O}(20 \mathrm{ml})$. The mixture was shaken at $0^{\circ} \mathrm{C}$ while $37 \%$ aq. $\mathrm{HCl}(2.6$ $\mathrm{mL}, 3.1 \mathrm{~mol}$ ) was added in portions over $10 \mathrm{~min}$. Then the slurry was shaken at $0 \mathrm{C}$ for a further $3 \mathrm{~h}$, filtered and washed with chilled water $(5 \times 20 \mathrm{ml})$. The resulting cellulose-bound diazonium salt 7 was transferred into water $(10 \mathrm{ml})$ at $0^{\circ} \mathrm{C}$. Then an ice water solution (15 ml) of $1 H$-pyrazole-1-carboxamidine hydrochloride $3(0.59 \mathrm{~g}, 4$ mmol $)$ and $\mathrm{NaHCO}_{3}(0.42 \mathrm{~g} 5 \mathrm{mmol})$ was added in portions at $0^{\circ} \mathrm{C}$ over $10 \mathrm{~min}$. The reaction mixture was shaken at $0^{\circ} \mathrm{C}$ for $3 \mathrm{~h}$ and for $12 \mathrm{~h}$ at room temperature. After 
filtration, the cellulose was washed with $\mathrm{H}_{2} \mathrm{O}(20 \mathrm{~mL})$ and stirred in EtOH $(20 \mathrm{~mL})$ for $1 \mathrm{~h}$, filtered again and washed with $\mathrm{EtOH}(20 \mathrm{~mL}), \mathrm{H}_{2} \mathrm{O}(20 \mathrm{~mL})$ and $\mathrm{EtOH}(20$ $\mathrm{mL})$. The cellulose 8 was then swollen in EtOH $(15 \mathrm{~mL})$. A few beads of cellulose were dried in high vacum for $1 \mathrm{~d}$ to yield pale brown beads of $\mathbf{8}$. Functionalization of all cellulose-bound amines was verified by the $\beta$-naftol test. ${ }^{2}$ The efficiency of the transformation according to nitrogen elemental analysis proved to be over $99 \%$. IR (KBr): 3228, 3097, 1675 (s), 1600, 1518, 1441, 1379, 1340(s), 1240, 1153 (s), 828

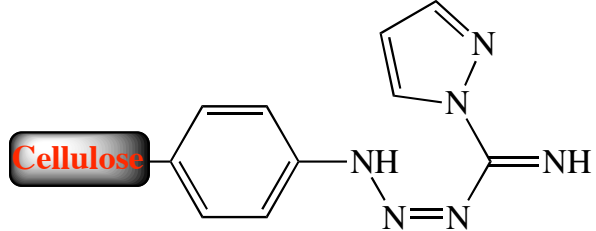

8

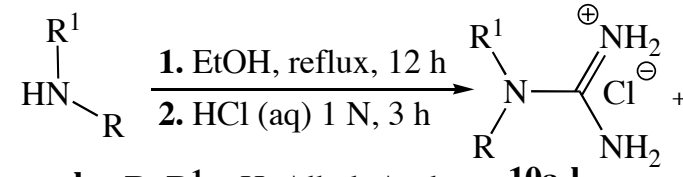

a-l $\quad \mathbf{R}, \mathbf{R}^{\mathbf{1}}=\mathrm{H}$, Alkyl, Aryl
10a-I

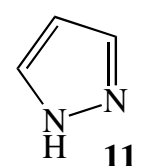

\section{General procedure to prepare guanidines 10a-l}

The cellulose 8 previously obtained was suspended in EtOH $(10 \mathrm{ml} / \mathrm{g}$ resin) and treated with the amine (5 equiv) and heated at reflux for $12 \mathrm{~h}$. The cellulose beads were filtered and then washed sequentially with EtOH, NMP, $2 \%$ aq. $\mathrm{NaHSO}_{4}, \mathrm{H}_{2} \mathrm{O}$, $\mathrm{EtOH}(3 \times 15 \mathrm{ml} / \mathrm{g}$ of cellulose), and dried in vacuum. The corresponding guanidine was cleaved from the polymer by treatment with $1 \mathrm{~N} \mathrm{HCl}$ in $\mathrm{H}_{2} \mathrm{O}(15 \mathrm{ml})$ under mechanical agitation for $3 \mathrm{~h}$. Subsequently; the cellulose was filtered and washed with $\mathrm{H}_{2} \mathrm{O}(2 \times 10 \mathrm{ml})$ and $\mathrm{EtOH}(15 \mathrm{ml})$. The cleavage step was repeated three times. The cellulose was then washed finally with $\mathrm{H}_{2} \mathrm{O}(2 \times 15 \mathrm{ml})$ and the combined filtrates were concentrated under vacuum to afford the desired guanidine $\mathbf{1 0}$ in yields up to $98 \%$.

(2) Gaggini, F.; Porcheddu, A.; Reginato, G.; Rodriquez, M.; Taddei, M. J. Comb. Chem. 2004; 6, 805-810. 
<smiles>CCCCNC(N)N</smiles>

n-Butylguanidine hydrochloride 10a. 98\% yield. Colourless oil. ${ }^{1} \mathrm{H}$ NMR (DMSO$\left.d_{6}\right) \delta 7.71(\mathrm{brs}, 1 \mathrm{H}), 7.15(\mathrm{brs}, 4 \mathrm{H}), 3.07(\mathrm{~m}, J=6.6,2 \mathrm{H}), 1.46(\mathrm{~m}, 2 \mathrm{H}), 1.34(\mathrm{~m}, 2 \mathrm{H})$, $0.86(\mathrm{t}, J=7.2 \mathrm{~Hz}, 3 \mathrm{H}) .{ }^{13} \mathrm{C}$ NMR (DMSO- $\left.d_{6}\right): \delta(\mathrm{ppm}) 158.4,37.9,33.4,20.1,14.3$. HPLC purity 99\%. (ESI + ve ion). Calcd for $\mathrm{C}_{5} \mathrm{H}_{14} \mathrm{~N}_{3}: 116.1\left[(\mathrm{M}+\mathrm{H})^{+}\right]$. Found: 116.1 . Anal. Calcd for $\mathrm{C}_{5} \mathrm{H}_{14} \mathrm{ClN}_{3}$ : C, 39.60; H, 9.31; N, 27.71. Found: C, 39.51; H, 9.26; N, 27.79 .

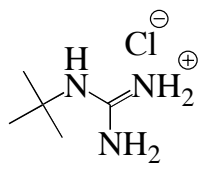

tert-Butylguanidine hydrochloride 10b. $91 \%$ yield. ${ }^{1} \mathrm{H}$ NMR (DMSO- $\left.d_{6}\right): \delta(\mathrm{ppm})$ $7.48(\mathrm{~s}, 1 \mathrm{H}), 7.00$ (brs, 4H), 1.29 (s, 9H). ${ }^{13} \mathrm{C}$ NMR (DMSO- $\left.d_{6}\right): \delta$ (ppm) 156.3, 51.5, 29.2. $\left(\mathrm{ESI}+\right.$ ve ion): Calcd for $\mathrm{C}_{5} \mathrm{H}_{14} \mathrm{~N}_{3}: 116.1\left[(\mathrm{M}+\mathrm{H})^{+}\right]$. Found: 116.1 . HPLC purity 99\%. Anal. Calcd for $\mathrm{C}_{5} \mathrm{H}_{14} \mathrm{ClN}_{3}$ : C, 39.60; H, 9.31; N, 27.71. Found: C, 39.68; H, $9.36 ; \mathrm{N}, 27.75$.

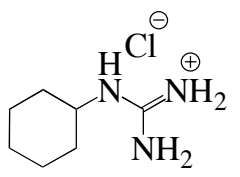

Cyclohexylguanidine Hydrochloride 10c. 95\% yield. white crystalline solid: 225$226{ }^{\circ} \mathrm{C}$ (litt. $\left.{ }^{3} 224-226\right) .{ }^{1} \mathrm{H}$ NMR (DMSO- $d 6$ ): $\delta(\mathrm{ppm}) 7.55$ (d, $\left.J=8.3 \mathrm{~Hz}, 1 \mathrm{H}\right), 6.63-$ 7.41 (brs, 4H), 3.45-3.30 (m, 1H), 1.74-1.89 (m, 2H), 1.60-1.71 (m, 2H), 1.50-1.58 
$(\mathrm{m}, 1 \mathrm{H}), 1.09-1.37(\mathrm{~m}, 5 \mathrm{H}),{ }^{13} \mathrm{C}$ NMR ((DMSO- $\left.d_{6}\right): 156.0,49.1,32.2,24.8,23.9$. $\left(\mathrm{ESI}+\right.$ ve ion). Calcd for $\mathrm{C}_{7} \mathrm{H}_{16} \mathrm{~N}_{3}: 142.1\left[(\mathrm{M}+\mathrm{H})^{+}\right]$. Found: $142.3\left[(\mathrm{M}+\mathrm{H})^{+}\right]$. HPLC purity 98\%. Anal. Calcd for $\mathrm{C}_{7} \mathrm{H}_{16} \mathrm{ClN}_{3}$ : C, 47.32; H, 9.08; N, 23.65. Found: C, 47.24; H, 9.17; N, 23.62.<smiles>NC(=[NH2+])NCc1ccccc1</smiles>

Benzylguanidine hydrochloride 10d. 98\% yield. White crystalline solid, mp 173$174^{\circ} \mathrm{C} .{ }^{1} \mathrm{H}$ NMR (DMSO- $\left.d_{6}\right): \delta(\mathrm{ppm}) 8.15$ (brs, $\left.1 \mathrm{H}\right), 6.84-7.62(\mathrm{~m}, 9 \mathrm{H}), 4.35$ (brs, 2H). ${ }^{13} \mathrm{C}$ NMR (DMSO- $\left.d_{6}\right): \delta(\mathrm{ppm}) 162.8,141.7,121.9,118.0,114.0,110.3$. (ESI + ve ion). Calcd for $\mathrm{C}_{8} \mathrm{H}_{12} \mathrm{~N}_{3}: 150.1\left[(\mathrm{M}+\mathrm{H})^{+}\right]$. Found: 150.2. HPLC purity 99\%. Anal. Calcd for $\mathrm{C}_{8} \mathrm{H}_{12} \mathrm{ClN}_{3}$ : C, 51.76; H, 6.51; N, 22.63. Found: C, 51.81; H, 6.44; N, 22.66.<smiles>CC(=O)NC(N)[NH3+]</smiles>

Methyl 2-(amidinoamino)acetate hydrochloride 10e. $65 \%$ yield. White hygroscopic foam. ${ }^{1} \mathrm{H}$ NMR (DMSO- $\left.d_{6}\right): \delta(\mathrm{ppm}) 7.45$ (s, 1H), 7.02 (brs, 4H), 3.58 (s, 3H), $3.21(\mathrm{~s}, 2 \mathrm{H}) .{ }^{13} \mathrm{C}$ NMR (DMSO- $\left.d_{6}\right): \delta(\mathrm{ppm}) 172.3,159.2,52.1,40.2$. (ESI + ve ion). Calcd for $\mathrm{C}_{4} \mathrm{H}_{10} \mathrm{~N}_{3} \mathrm{O}_{2}: 132.1\left[(\mathrm{M}+\mathrm{H})^{+}\right]$. Found: 132.3. HPLC purity 97\%. Anal. Calcd for $\mathrm{C}_{4} \mathrm{H}_{10} \mathrm{ClN}_{3} \mathrm{O}_{2}$ : C, 28.67; H, 6.01; N, 25.07. Found: C, 28.71; H, 6.13; N, 25.02 .<smiles>NC(Nc1ccccc1)N(N)Cl</smiles>

Phenylguanidine hydrochloride 10f. $71 \%$ yield. ${ }^{1} \mathrm{H}$ NMR (DMSO- $\left.d_{6}\right): 7.24$ (d, 
$J=7.4 \mathrm{~Hz}, 2 \mathrm{H}), 7.29$ (t, $J=7.4 \mathrm{~Hz}, 1 \mathrm{H}), 7.45$ (t, $J=7.9 \mathrm{~Hz}, 2 \mathrm{H}), 7.61$ (brs, 4H), 10.07 (brs, $1 \mathrm{H}) .{ }^{13} \mathrm{C}$ NMR ((DMSO- $\left.d_{6}\right): \delta(\mathrm{ppm}) 158.2,142.6,129.2,128.8,118.3,115.7$. (ESI + ve ion). Calcd for $\mathrm{C}_{7} \mathrm{H}_{10} \mathrm{~N}_{3}: 135.1\left[(\mathrm{M}+\mathrm{H})^{+}\right]$. Found: $135.2\left[(\mathrm{M}+\mathrm{H})^{+}\right]$. HPLC purity 97\%. Anal. Calcd for $\mathrm{C}_{7} \mathrm{H}_{10} \mathrm{ClN}_{3}$ : C, 48.99; H, 5.87; N, 20.66. Found: C, 48.90; H, 5.79; N, 20.67.<smiles></smiles>

(4-Methoxyphenyl)guanidine hydrochloride 10g. $78 \%$ yield. white crystalline solid: mp 143-144 ${ }^{\circ} \mathrm{C}$ (lit. $\left.{ }^{4} \mathrm{mp} 144-146{ }^{\circ} \mathrm{C}\right) .{ }^{1} \mathrm{H}$ NMR (DMSO-d 6 ): $\delta$ (ppm) 8.37 (brs, 1H), 7.36 (broad s, 4H), $7.18(\mathrm{~d}, J=8.9 \mathrm{~Hz}, 2 \mathrm{H}), 7.00(\mathrm{~d}, J=8.9 \mathrm{~Hz}, 2 \mathrm{H}), 3.77$ (s,

$3 \mathrm{H}) .{ }^{13} \mathrm{C}$ NMR (DMSO- $\left.d_{6}\right): \delta(\mathrm{ppm}) 158.5,150.1,136.9,117.9,115.6,61.1 .(\mathrm{ESI}+\mathrm{ve}$ ion). Calcd for $\mathrm{C}_{8} \mathrm{H}_{12} \mathrm{~N}_{3}: 166.1\left[(\mathrm{M}+\mathrm{H})^{+}\right]$. Found: 166.3. HPLC purity 98\%. Anal. Calcd for $\mathrm{C}_{8} \mathrm{H}_{12} \mathrm{C} 1 \mathrm{~N}_{3} \mathrm{O}: \mathrm{C}, 47.65 ; \mathrm{H}, 6.00 ; \mathrm{N}, 20.84$. Found: C, 47.59; H, 5.97; N, 20.90 .

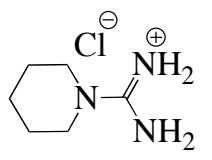

Piperidine-1-carboxamidine hydrochloride 10i. 93\% yield. White solid, mp 186-87 ${ }^{\circ} \mathrm{C}$, (lit. $\left.{ }^{5} \mathrm{mp} 187-188{ }^{\circ} \mathrm{C}\right) ;{ }^{1} \mathrm{H}$ NMR (DMSO- $\left.d_{6}\right): \delta(\mathrm{ppm}): 5.2-4.3$ (br s, 3H), $3.31(\mathrm{t}$, $J=5.25 \mathrm{~Hz}, 4 \mathrm{H}), 1.54-1.37(\mathrm{~m}, 6 \mathrm{H}) .{ }^{13} \mathrm{C}$ NMR (DMSO-d6): $\delta(\mathrm{ppm}): 158.8,45.9$, 25.1, 24.0. (ESI + ve ion). Calcd for $\mathrm{C}_{6} \mathrm{H}_{14} \mathrm{~N}_{3}: 128.1\left[(\mathrm{M}+\mathrm{H})^{+}\right]$. Found: 127.9. HPLC

(4) Bernatowicz, M. S.; Wu, Y.; Matsueda, G. R. J. Org.Chem. 1992, 57, 2497-2502.

(5) Bannard, R. A. B.; Casselman, A. A.; Cockburn, W. F.; Brown, G. M. Can. J. Chem. 1958, 36, 1541. 
purity 98\%. Anal. Calcd for $\mathrm{C}_{6} \mathrm{H}_{14} \mathrm{ClN}_{3}$ : C, 44.04; H, 8.62; N, 25.68. Found: C, 44.12; H, 8.70; N, 25.57.

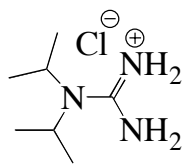

Diisopropylguanidine hydrochloride 101. 91\% yield. White hygroscopic foam. ${ }^{1} \mathrm{H}$ NMR (DMSO-d $\left.)_{6}\right): \delta(p p m) 7.03($ br s, 4H), $3.93(\mathrm{~h}, J=6.8 \mathrm{~Hz}, 2 \mathrm{H}), 1.24$ (d, $J=6.8$ $\mathrm{Hz}, 12 \mathrm{H}) .{ }^{13} \mathrm{C}$ NMR (DMSO- $\left.d_{6}\right): \delta(\mathrm{ppm}): 158.2,43.4,21.8$. (ESI + ve ion). Calcd for $\mathrm{C}_{7} \mathrm{H}_{8} \mathrm{~N}_{3}: 143.1\left[(\mathrm{M}+\mathrm{H})^{+}\right]$. Found: 143.2. $\left[(\mathrm{M}+\mathrm{H})^{+}\right]$. HPLC purity $97 \%$. Anal. Calcd for $\mathrm{C}_{7} \mathrm{H}_{8} \mathrm{ClN}_{3}$ : C, 46.79; H, 10.10; N, 23.38. Found: C, 46.85; H, 10.01; N, 23.27. 